\title{
NanoElectrocatalysis: From Basic Research to Applications in Energy Conversion
}

\author{
Matthias Arenz ${ }^{\star}$
}

\begin{abstract}
In this article an overview of our research in the field of nanoelectrocatalysis, in particular electrocatalytic energy conversion, is presented. Our research comprises the areas of catalyst preparation as well as performance evaluation of model and applied electrocatalysts with respect to activity, selectivity, and stability. Our aim is to understand the fundamental properties that determine the performance of electrocatalysts for particular processes and to build a bridge between fundamental research and realistic applications. In our research we prepare catalyst materials, study the interface between the catalyst and the electrochemical environment, i.e. the solid-liquid interface, and perform kinetic measurements. Furthermore, such research relies to a large extent on material characterization techniques as well as the development and application of advanced experimental setups.
\end{abstract}

Keywords: Catalyst preparation - Energy conversion $\cdot$ In situ characterization $\cdot$ NanoElectrocatalysis $\cdot$ Solidliquid interface

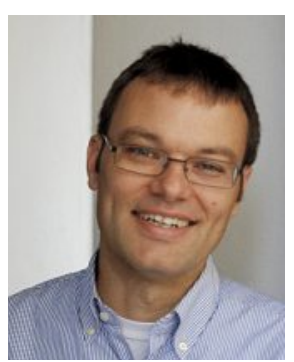

lishing in Munich (2006) an independent Emmy Noether Group of the German Science Foundation (DFG). In 2010 he became tenured Associate Professor at the University of Copenhagen (Denmark) and in 2016 Full Professor at the University of Bern (Switzerland). His group focusses on electrocatalytic reactions related to energy conversion and storage.

Matthias Arenz studied physics with chemistry minor in Bonn (Germany) and received his diploma (Physical Chemistry) in 1999 and in 2002 his $\mathrm{PhD}$ on model electrodes for electrocatalysis with Prof. K. Wandelt. Already during his $\mathrm{PhD}$ he spent seven months in the group of Dr. P. N. Ross and Dr. N. M. Markovic at the Lawrence Berkeley National Laboratory (USA) and returned to the group in 2002 with a Feodor Lynen Fellowship (A.v. Humboldt Foundation) for two years of postdoctoral work. Back in Germany, he joined in 2004 the group of Prof. U. Heiz in Ulm and Munich, before estab-

${ }^{\star}$ Correspondence: Prof. M. Arenz Department of Chemistry and Biochemistry University of Bern

Freiestrasse 3, $\mathrm{CH}-3012$ Bern

E-mail: matthias.arenz@dcb.unibe.ch

\section{Introduction}

Our research focuses on electrocatalytic energy conversion, i.e. the conversion of energy stored in chemical bonds into electricity as well as the reverse process, the conversion of electricity into chemicals. These chemicals might serve as fuels (energy carriers) but can also be high value chemicals. The motivation of such research is strongly connected to the concept of sustainable chemistry. Today most chemicals and fuels are derived from fossil resources, which are limited. Moreover, their ongoing recovery and consumption leads to significant ecological challenges. Therefore, fossil resources must be stepwise replaced by sustainable sources such as biomass, wind, and solar power. The latter two are intermittent energy sources that 'produce' electricity. To align energy 'production' from these intermittent energy sources and the demand from the user, we need processes that can store electricity, processes that convert stored electricity back to electric power, but also processes that convert electricity into chemicals using abundant feedstocks. In all these processes catalysts, in particular electrocatalysts, play a key role. By offering alternative reaction pathways of reduced energy barrier (see scheme in Fig. 1), catalysts enhance the reaction rate, control the selectivity and thus render processes more efficient.

In our research we try to understand such energy conversion processes and to develop materials that help to facilitate them. Our vision thereby is to bridge the gap between fundamental and applied research. Therefore, we conduct studies on so-called model catalysts as well as industrial type catalysts. This research is interdisciplinary combining aspects of chemistry, surface science, and spectroscopy, but also engineering. The basis for developing improved catalyst materials is a sound concept for catalyst preparation. We concentrate on two approaches, one to develop supported high surface area catalysts; an approach we dubbed the tool-box approach to highlight the idea to systematically control the individual catalyst parameters independent of each other. The second approach is to prepare supportfree, nanoporous metal networks, which combines bulk-like properties with high surface area and therefore is suitable for precious metals as well.

The basis for understanding catalytic processes are advanced experimental techniques and protocols. These include in situ and online methodologies such as differential electrochemical mass spectrometry (DEMS), Fourier-transform infrared spectroscopy (FTIR) and X-ray absorption spectroscopy (XAS); but also the design of electrochemical cells with improved 
Fig. 1. Sketch of working principle of a catalyst.

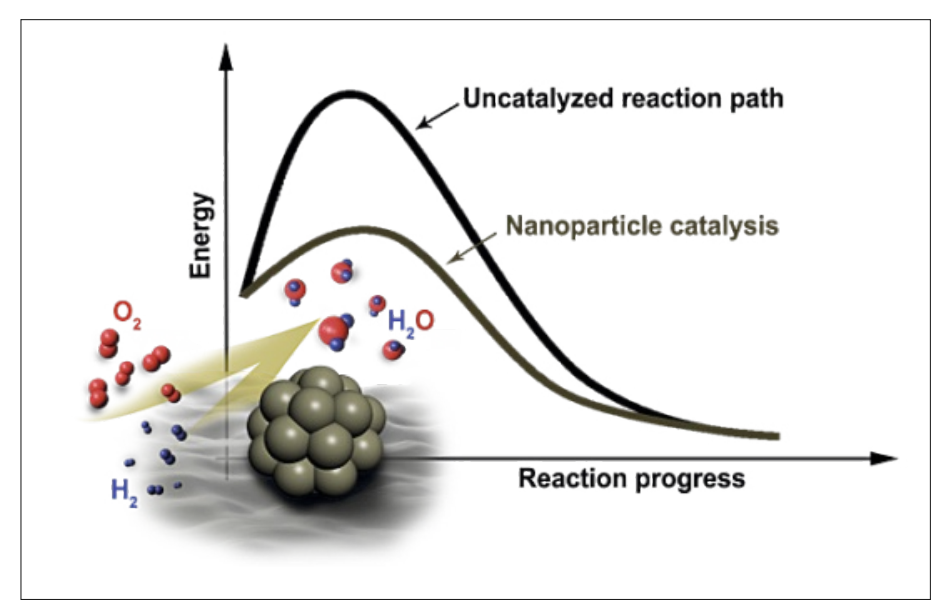

mass transport and experimental protocols to follow catalyst degradation or to better probe the solid-liquid interface. Our general research strategy is not to concentrate on a single investigation method, but to combine the results of as many techniques as possible and to develop and improve techniques based on specific requirements. Last but not least, combining experimental work with computational simulations is a fruitful combination, which we pursue in collaborative work.

In the following, we first introduce our concept for catalyst preparation. In the next chapter, we introduce some experimental techniques and protocols our group made significant contributions to develop, followed by case examples of our research.

\section{Catalyst Preparation}

In heterogeneous catalysis, tailor-made catalysts are often referred to. The idea is that catalysts are designed according to specific, known design parameters that guarantee an optimized performance for a specific application. General design strategies have been derived from surface science and studies on so-called model catalysts, i.e. catalysts that have very defined properties, but cannot be applied in a technical process. These studies significantly improved our general knowledge in structure-function relationships of catalysts. Nevertheless, there is a gap between this general knowledge and how catalysts are developed in industry. In industry catalyst screening-based approaches are still common and often the most efficient way to optimize a catalyst. A famous example from the past in this regard was the Haber-Bosch process of ammonia production, which had been applied for several decades before resolving the reaction mechanism. ${ }^{[1]}$ In part, this gap between application and understanding ${ }^{[2]}$ is due to the tremendous complexity of catalytic processes. In part however, this is also due to a lack of suitable preparation strategies that can use the knowledge we have to synthesize applied catalysts according to specific design criteria. Standard catalyst preparation protocols do not allow for adjusting independently important catalyst properties. This and the fact that catalysts change during operation, and as a consequence what is characterized after catalyst preparation is rather a precursor than the active catalyst, inhibit a true materials-by-design approach. Preparing catalysts is therefore often referred to as a 'black art'. [3] These limitations in catalyst preparation are not only unsatisfactory from an applied point of view, but even more so from a scientific point view. Scientific research requires designing experiments where only a single, but not multiple, parameters are changed at once to support or disprove a hypothesis. This fundamental concept of scientific work is surprisingly often ignored in academic catalysis research.

In the following, our approach of a tailored catalyst preparation for systematic studies is discussed. The change of catalysts during operation is addressed in later sections. One can classify catalysts into supported high surface area and bulk-type catalysts. Supported high surface area catalysts consist of a highly dispersed active phase and a support material. Supported high surface area catalysts are in general used if the active catalyst material is expensive, e.g. if it consists of precious group metals (PGMs). A typical example are $\mathrm{Pt}$ and Pt-alloy nanoparticles supported on a high surface area carbon, see below. Bulktype catalysts are unsupported and are more common for processes where the active catalyst material is neither rare nor expensive. The best known example of a bulk catalyst is Raney nickel, which is skeletal nickel derived from a nickel-aluminum alloy by de-alloying. [4]

First, we discuss our concept for the preparation of supported catalysts for systematic studies, then support-free, nanoporous metal networks are introduced as a concept for bulk-type catalysts consisting of PGMs.

\subsection{Colloidal Tool-box Synthesis of Supported High Surface Area Catalysts}

An example for supported high surface area catalysts are state-of-the-art proton exchange membrane fuel cell (PEMFC) catalysts. They consist of an active phase of Pt or Pt-alloy nanoparticles that are distributed over a high surface area carbon support. The active nanoparticles catalyze the reactions (oxygen reduction on the cathode and hydrogen oxidation on the anode), whilst the carbon support not only keeps the active phase dispersed, it also conducts the involved electrons and guarantees sufficient reactant transport. From a simplified point of view only the catalyst performance (activity, selectivity, and stability) and the material costs (mainly the precious metal content) are important. For the catalyst activity, this property is expressed by the mass activity. However, manufacturing cost, material abundance (related to the cost), and reproducibility in the preparation method are important considerations as well if a catalyst is to be used in an industrial process. From a scientific viewpoint, it is also essential to be able to conduct systematic investigations with full control over the catalyst properties to optimize a catalyst, but also to discover phenomena and mechanistic aspects. In an example introduced below concerning the particle size effect of the oxygen reduction reaction (ORR) usually several parameters are varied at once (in particular particle size and interparticle distance) thus inhibiting to address observed effects to a single catalyst property.

Industrial synthesis methods for electrocatalysts are based on impregnation and incipient wetness techniques to cover the support with the metal salt precursor, followed by calcination and reduction treatments that lead to the formation of the active phase in form of nanoparticles. ${ }^{[5]}$ These methods allow large-scale production, but they lack the possibility to change individual catalyst properties independently, e.g. particle size and metal loading cannot be varied independent of each other. The main reason for these limitations in catalyst preparation is that the active phase is directly formed on the carbon support. As a result, the formation of the active phase depends strongly on the support properties. Posttreatment may not only influence the active phase but also the support. Finally, the accessibility of the active phase for the reactants might be limited. Due to capillary forces particles might be formed in small pores, which inhibits reactant mass transport.[6]

Natural science is an empirical science. Hypotheses generally start with phenomenological discoveries. An important aspect in this regard is to test hypotheses 
in a suitable manner. Often this is limited not only by the available investigation techniques, but also by catalyst preparation. As outlined above, we aim to perform hypothesis-driven research. In order to be able to test certain hypotheses, in recent years our research group developed, in collaboration with University of Bremen, a colloidal tool-box synthesis platform for electrocatalysts that provides high control and flexibility over the individual catalyst parameters. ${ }^{\text {[7] }}$ The 'tool-box' synthesis approach is based on a two-step preparation protocol. First, colloidal nanoparticles of well-defined size and composition are prepared, which in a second step are deposited onto a support. This approach disentangles the formation of the active phase from the specific support material and type. The benefits are that parameters like particle size, particle composition, and particle loading (on support) can be optimized/ varied independent of each other and independent of the support type. Furthermore, nanoparticles inside inaccessible pores of the support can be avoided and no posttreatment is needed that may irreversibly alter the support properties.

For the preparation of colloidal nanoparticles, we adopted the alkaline polyol approach presented by Wang et al. ${ }^{[8]}$ The method uses alkaline ethylene glycol (EG) as solvent and reducing agent, whereas the $\mathrm{OH}^{-}$is needed for obtaining stable colloids. ${ }^{[9]}$ In contrast to the majority of colloidal syntheses approaches no additional surfactants, ligands, or stabilizing agents such as polyvinylpyrrolidone (PVP) are needed. Therefore, Wang et al. referred to these colloidal nanoparticles as 'unprotected' ${ }^{[10]}$ As this term might be misleading, in the following we address these colloids as 'surfactant-free', fully being aware that their surface is actually covered by species protecting them from sintering. ${ }^{[9,11]}$ In our work concerning the colloidal stability of nanoparticles prepared by the alkaline EG approach, it could be shown that the nanoparticles are mainly protected by $\mathrm{OH}^{-}$and $\mathrm{CO}$ groups, which both can be removed easily by washing with $\mathrm{H}_{2} \mathrm{O}$ without irreversibly affecting the nanoparticles. ${ }^{[9,11 a, 12]}$ Alternatively, ligands can be bound to these nanoparticles while the particle size is maintained. ${ }^{[8,13]}$ This allows for systematic investigations concerning the effect of ligands on the catalytic properties of nanoparticles which can even lead to promoting effects as demonstrated by the above described strategy. ${ }^{[14]}$ In general, however, ligands inhibit catalytic processes as they block surface atoms and hence need to be removed prior to any application. This is in particular the case for strongly binding ligands such as PVP, which ensure very good colloidal stability against agglomeration or sintering, but at the cost of influenc- ing catalytic reactions in an unknown manner. Therefore, they need to be removed from the catalyst surface, e.g. by ozone treatment before the catalytic measurements. ${ }^{[15]}$ Nevertheless even by such harsh treatments protecting surfactants are not completely removed, leading to undesired changes in the catalytic properties and/or loss in surface area, as well as alteration of the support properties. ${ }^{[16]}$

The basic synthesis scheme for preparing supported catalysts from 'surfactantfree' colloids is sketched in Fig. 2. Preparing metal nanoparticles in alkaline EG synthesis using a microwave reactor allows very high reproducibility and reduces the time to prepare a stable colloidal nanoparticle suspension to just a few minutes. ${ }^{[7 b, 17]}$ Within a day, literally dozens of different colloid samples can be produced. To prepare a sup- ported catalyst, the nanoparticles need to be precipitated with $\mathrm{HCl}$ (often referred to as 'washing'), redispersed in an organic solvent with a low boiling point (e.g. acetone) from which they can be deposited onto support by simply drying. These procedures, i.e. the colloidal Pt nanoparticle preparation and supporting step are described in detail in a recent open access tutorial ${ }^{[17 a]}$ and thus will not be described here further. It is always accompanied by a proper characterization of obtained catalyst samples, e.g. by transmission electron microscopy (TEM) and/or small angle X-ray scattering (SAXS). Furthermore, the obtained metal loading on the support needs to be determined by complete digestion in aqua regia and inductively coupled plasma mass spectrometry (ICP-MS) or thermogravimetric analysis (TGA).

\section{NP synthesis}

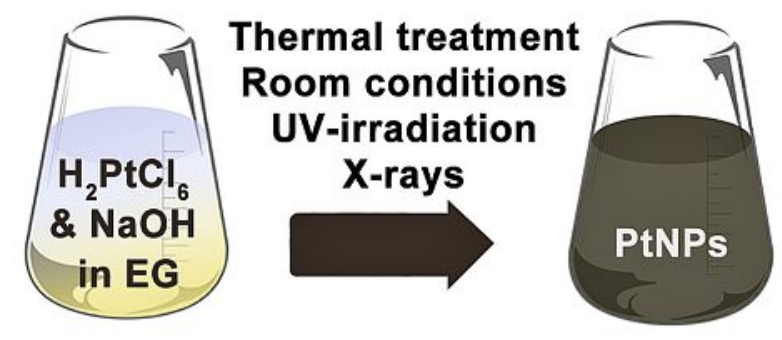

\section{Catalyst formation}

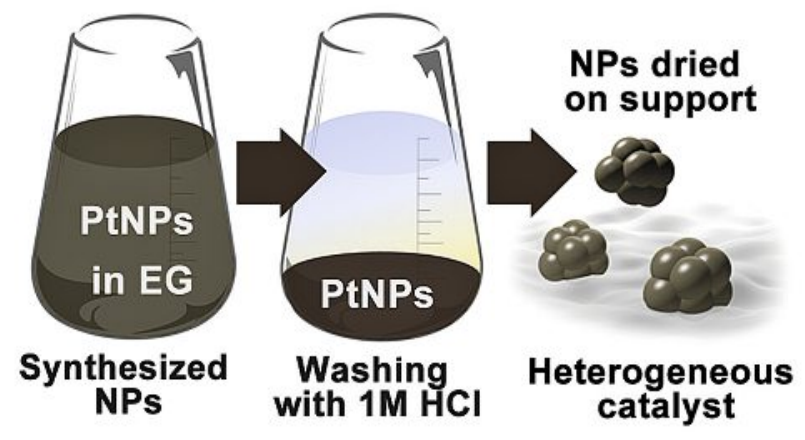

\section{Independent control on:}

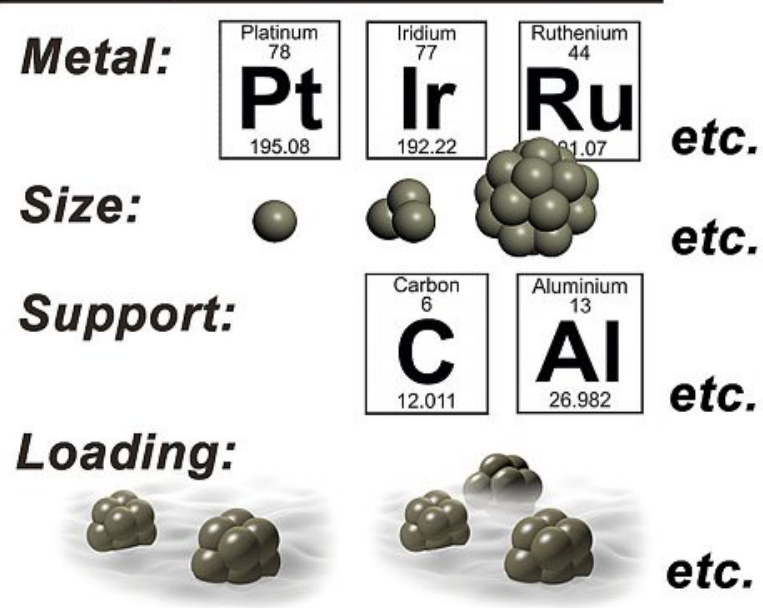

Fig. 2. Sketch of the tool-box approach based on the colloidal synthesis of precious metal nanoparticles in alkaline ethylene glycol. 
In previous studies, the 'tool-box' approach was used to study the particle proximity effect on the ORR as well as the influence on the $\mathrm{Pt}$ loading and support type on the stability of PEMFC catalysts, see below. More recently, we significantly improved the general synthesis approach by replacing EG. The use of EG as solvent requires its removal by a precipitation step with $1 \mathrm{M} \mathrm{HCl}$, as already described in the original work by Wang et al., ${ }^{[8]}$ before redispersion of the nanoparticles and their deposition onto a support material. As chlorides are a severe catalyst poison, this step is not uncritical. Investigating small amounts of catalyst, the chlorides can be removed by washing the catalyst powder in deionized (DI) water. Followed by a $\mathrm{pH}$ adjustment of the catalyst ink, highly performing catalyst layers can be produced. ${ }^{[18]}$ In addition, we investigated different alternatives to $\mathrm{HCl}$, with some promising results. Nevertheless, all these steps increase the amount of waste produced in the catalyst preparation, which diminishes its potential for production at larger scale. Therefore, we intensively searched for strategies to prepare colloidal nanoparticles without the use of surfactants that in contrast to the alkaline EG method exhibit chemical properties that allow for completely avoiding any precipitation and post-synthetic treatments to prepare supported catalysts. We successfully achieved a synthesis approach for nanoparticles in low boiling point solvents such as methanol or ethanol without the use of surfactants like polymers. ${ }^{[19]}$ Particle size and composition can be tuned by adjusting the synthesis conditions. Colloids synthesized by our low boiling point solvent approach can be mixed with the support and then be deposited by simple drying. Thus obtained catalysts can be washed, preferably with protic solutions that may be organic (e.g. trifluoroacetic acid, formic acid, acetic acid, etc.) or aqueous solutions $\left(\mathrm{HNO}_{3}\right.$, $\mathrm{H}_{2} \mathrm{SO}_{4}, \mathrm{H}_{3} \mathrm{PO}_{4}$, oxalic acid, etc.) to remove synthetic residues. Unlike catalysts prepared by other approaches, no further post-synthetic steps are needed to activate these nanoparticles.

\subsection{Support-free, Nanoporous Metal Networks}

As discussed above, supported high surface area catalysts are commonly used in cases where the active material is rare and expensive. How effective the material is used is displayed in the electrochemically accessible surface area (ECSA). The ECSA strongly depends on the particle size. For Pt-based PEMFC catalysts values range from $140 \mathrm{~m}^{2} \mathrm{~g}_{\mathrm{Pt}}{ }^{-1}$ down to less than $40 \mathrm{~m}^{2} \mathrm{~g}_{\mathrm{Pt}}{ }^{-1}$. Maximizing the ECSA might negatively affect the catalyst stability as small nanoparticles are less stable than larger ones. In addition, for supported high surface area catalysts, support corrosion needs to be considered; especially in case of carbon supports which have a very limited stability (potential) window and are only kinetically stable at applied conditions.

Therefore, several approaches have been presented to avoid the use of carbon supports for electrocatalysts that experience high potentials under operation, e.g. ORR catalysts. $3 \mathrm{M}$ demonstrated nanostructured thin film (NSTF) ORR catalysts, where the carbon support has been replaced by a non-conducting inorganic dye (perylene red whiskers). ${ }^{[20]}$ The advantages are high specific activities (SA) and good degradation resistance, however, at the expense of the ECSA. For NSTF PEMFC catalysts ECSA values below $10 \mathrm{~m}^{2} \mathrm{~g}_{\mathrm{Pt}}{ }^{-1}$ are reported. [21] Another example of unsupported ORR catalysts are Pt-alloy aerogels, which can achieve considerably higher ECSA values $\left(c a .30 \mathrm{~m}^{2}\right.$ $\left.\mathrm{g}_{\mathrm{Pt}}{ }^{-1}[22]\right)$, while maintaining improvements in SA and durability.

In collaboration with the Leibniz Institute for Plasma Science and Technology (INP), we developed an alternative approach of support-free, nanoporous thin metal networks, see Fig. 3. The basic idea is to use alternating magnetron sputtering of layers of a noble metal, e.g. Pt for PEMFC applications, and a second, less (non) noble component, e.g. $\mathrm{Co}$ or $\mathrm{Cu}$, to create a mixed metal film. The non-noble component thereby is sputtered at large excess, e.g. in the case of $\mathrm{Pt}$ and Co ratios of $1: 6$ up to $1: 14$ were applied. The substrate may be a glassy carbon (GC) electrode for measurements in electrochemical half-cells, or directly a gas diffusion layer (GDL) such as applied in fuel cell systems. If beneficial for alloying, a subsequent heat treatment can be applied. In the next step, the non-noble component can be leached out of the metal film by a simple acid treatment. This step can be done with or without potential control. Leaching out the excess non-noble component a nanoporous metal network is created that combines the favorable properties of bulk-type catalysts with exceptional high surface areas. Studies to apply Pt-based nanoporous networks as PEMFC catalysts are currently in progress, however, such nanoporous metal networks can be applied in other areas as well.

\section{Method Developments}

Equally important as catalyst preparation are suitable experimental methods and protocols for evaluating catalyst performance. In this regard in situ and online methods are of particular importance for our research. Also, automation of electrochemical measurements and the coupling of different devices via LabView was a cornerstone of our work that eventually led to the founding of the spin-off company NordicElectrochemistry. ${ }^{[23]}$ The basic principles of in situ methods can be found in many advanced textbooks, therefore in the following only those methods and procedures are introduced and discussed, where our research group made significant contributions to their development.

\subsection{Identical-location Microscopy for Studying Catalyst Degradation}

The stability of a catalyst is of crucial importance for its applicability. Even highly active catalysts cannot be used if they do not exhibit sufficient lifetime. There are different approaches to investigate catalyst stability. Probing the activity of a catalyst upon specific accelerated degradation tests (ADTs) provides information if a catalyst is stable or not under these specific conditions. Often such measurements are used to 'prove' that a catalyst indeed is stable, while conditions where the catalyst is instable are avoided. Such studies do not provide any scientific insight into the "mechanistics' of degradation processes. Thus, the acquired knowledge is limited and does not promote the development of catalysts with

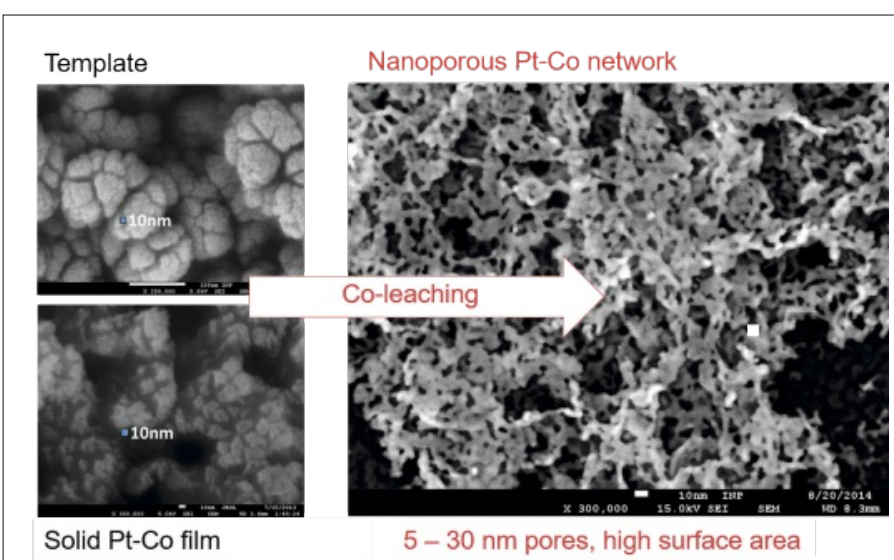

Fig. 3. Preparation of nanoporous Pt-Co film via acid leaching of Co.

5 - $30 \mathrm{~nm}$ pores, high surface area 
improved properties. Further, the information is limited to specific reaction conditions. This is meaningful if it should be decided which catalyst is most suitable for a very specific, defined process. In electrocatalytic processes, however, the reaction conditions are often not (yet) completely defined and it is essential to investigate how the catalyst reacts to different experimental protocols, i.e. where the 'boundaries' of stability are. No catalyst is stable under all conditions. From a scientific point of view it is of fundamental importance to understand specific degradation mechanisms and how they depend on specific operation conditions. For this electrocatalysts need to be exposed to conditions where performance degradation is observed.

In studies of supported high surface area catalysts, transmission electron microscopy (TEM) is a popular method. Establishing particle size histograms of the catalyst before and after a treating the catalyst with ADTs in principle can provide insights which characteristic degradation mechanism is predominant, i.e. particle migration and coalescence, metal dissolution, (electrochemical) Ostwald ripening, or particle detachment, see also below. However, these studies are limited by the fact that supported high surface area catalysts are in general quite inhomogeneous and TEM studies are always selective, even if different areas of the catalyst are investigated. We introduced the identical-location (IL) electron microscopy concept to alleviate these limitations in TEM degradation studies, see scheme in Fig. 4.24] The basic idea is to attach a thin film of high surface area catalyst onto a so-called TEM-finder grid, which then is used as a working electrode in ADTs. Probing identical locations of the catalyst layer before and after the ADT, the degradation mechanism can be followed on a selected number of individual particles. This technique significantly improves our understanding of degradation mechanisms and has been widely adopted and developed further for applications in electro- and gas-phase catalysis by many other research groups. ${ }^{[25]}$ In examples from our group, with the help of IL-TEM it could be shown that for a specific catalyst and treatment particle detachment was the main degradation channel, ${ }^{[24 a]}$ or that the treatment of a carbon support led to a significantly reduced loss mechanism, while not avoiding particle coalescence, see also below. ${ }^{[24 c]}$

\subsection{In situ FTIR Spectroscopy for Electrocatalytic Studies}

Fourier-transform infrared spectroscopy (FT-IRS) is an effective tool to investigate the behavior of molecules at the solid-liquid interface. The molecules of interest can be reactants and intermediates,

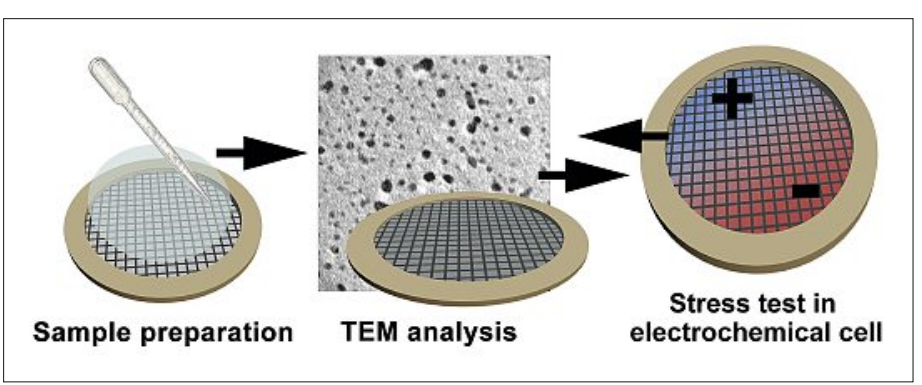

Fig. 4. Sketch of the IL-TEM concept for probing identical particles before and after degradation tests.

but also so-called spectator species from the electrolyte. The combination of IRS with electrochemical control poses some challenges, in particular when working with aqueous-based electrolytes, which exhibit high IR absorption. There are two main experimental configurations for the coupling of IRS to electrochemistry, i.e. infrared reflection absorption spectroscopy (IRAS) and the attenuated total reflection (ATR) configuration. The IRAS configuration is suitable when working with reflective electrode materials, but it has the inherent disadvantage that a confined space between a IR transparent prism and the working electrode is created that strongly inhibits any mass transport to the electrode surface. IRAS is therefore most suitable for the investigation of anion adsorption ${ }^{[26]}$ or processes, where a change of a pre-adsorbed layer of molecules is detected on the electrode such as in $\mathrm{CO}$ oxidation studies. ${ }^{[27]}$

In the ATR configuration the working electrode is directly attached to an ATR prism and therefore the electrolyte above the electrode is fully accessible. Molecular vibrations are detected via the so-called evanescent wave 'leaking' into the solidliquid interface. Therefore, the method is much more flexible for investigating dynamic changes at the solid-liquid interface than IRAS. Electrochemically coupled ATR-FTIRS is also known under the name surface-enhanced infrared absorption spectroscopy (SEIRAS). The method has been developed by Osawa. ${ }^{[28]}$ Our contribution to this field is the further development by coupling ATR-FTIR to an electrochemical flow cell[29] as well as introducing a 'finger-type' electrode that allows the study of supported high surface area catalysts. ${ }^{[30]}$ The coupling to an electrochemical flow cell was an important step to investigate the influence of anions on catalytic reactions such as the ORR. In previous investigations, the results from kinetic measurements were always compared to anion adsorption investigations performed on different electrodes in different electrochemical cells. This significantly limits the validity of the measurements as compared to the case where the reaction kinetics and the FTIR spectra are recorded simultaneously. The main experimental difficulty in our development was to obtain stable electrode layers onto an ATR prism, which can consist of silicon, germanium or zinc selenide. Poor adhesion of the electrode layers on the ATR prism has been reported in static cells. ${ }^{[31]}$ However, adhesion is especially challenging for the coupling with flow cells where electrolyte convection induces strong friction often leading to a lifting of the electrode layer on the prism. The use of adhesion layers of chromium or titanium followed by an 'activation treatment' has been reported in literature, but we could show that these layers render the electrode layer non-transparent for the IR beam. ${ }^{[29,30]}$ Modifying the ATR prism with a $\mathrm{Cr}$-Au layer in a 'finger' pattern provided a clever solution to the problem. The $\mathrm{Cr}$ adhesion layer fixed the gold layer, which is also used for electrical contact, strongly to the ATR prism. The patterned electrode can be spray-coated with any high surface area catalyst; the space between the 'fingers' is large enough for the IR beam to penetrate the catalyst layers and at the same time allows electrochemical contact to the gold layer (see Fig. 5). As the Cr$\mathrm{Au}$ is non-transparent for the IR beam, no information from the interface with the $\mathrm{Au}$ layer disturbs the analysis. Thus high surface area catalysts can be investigated in an ATR configuration coupled to a flow cell setup. ${ }^{[30]}$

\subsection{Experimental Setups for Im- proved Mass Transport}

The standard method for examining the kinetic reaction rate of an electrocatalyst are thin-film rotating disk electrode (TFRDE) measurements. ${ }^{[32]}$ The method was derived from RDE measurements using flat, bulk metal disk electrodes. Inserting the disk electrode into a tip of inert material, e.g. polytetrafluoroethylene (Teflon), and rotating the electrode around its own axis, allows a homogeneous and controlled mass flow of the reactants (dissolved) in the electrolyte to the electrode surface. From hydrodynamic equations, the measured current density (reaction rate) can be divided into a kinetic and a mass transport term. In the TF version of the RDE methodology, the disk electrode is an inert glassy carbon (GC) electrode covered by 


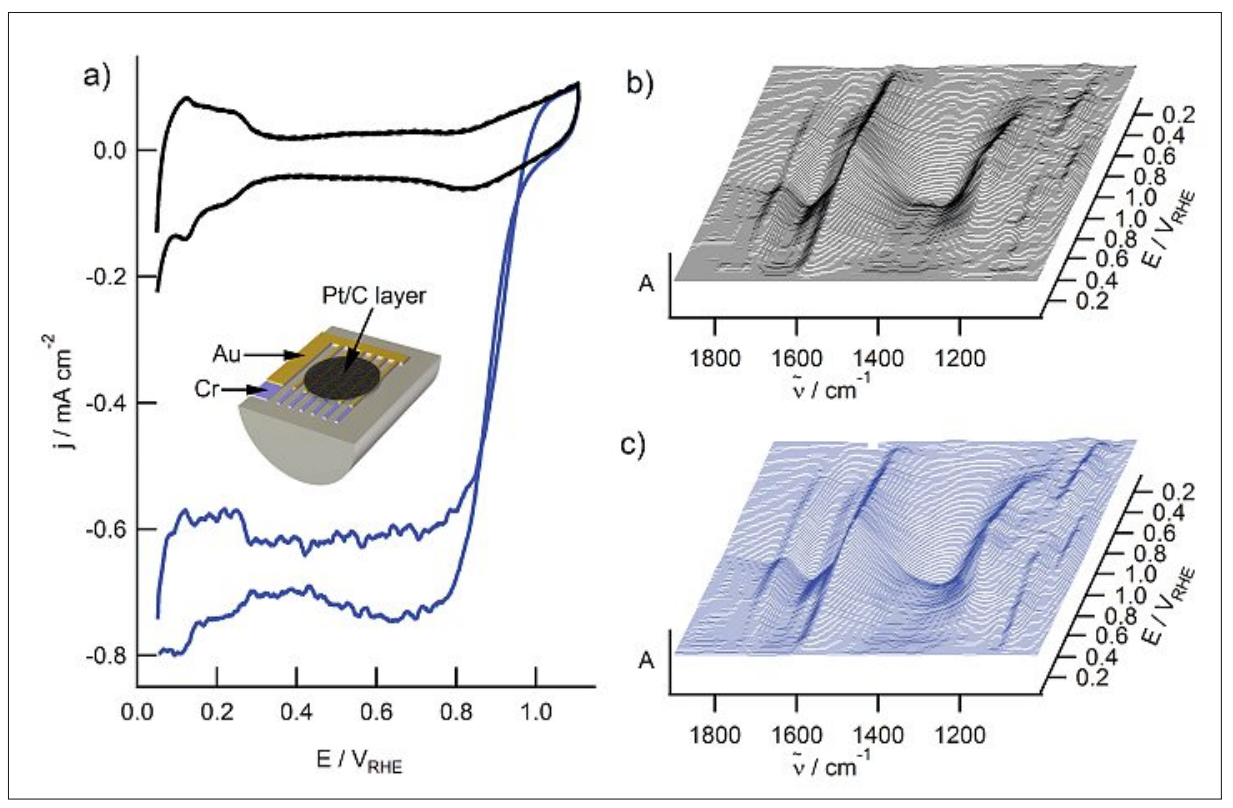

Fig. 5. Application of the finger-type electrode for simultaneously recording electrochemical polarization curves and in situ FTIR spectra. Figure reproduced with permission of Wiley- $\mathrm{VCH}^{\left[{ }^{[3]}\right]}$

a very thin film of supported high surface area catalyst.

The TF-RDE method is extensively used to determine catalyst activities, which can be expressed as the reaction rate at a certain electrode potential normalized to the electrochemical surface area (specific activity; SA) or the precious metal mass (mass activity; MA), respectively. The method is fast and requires only small amounts of catalyst material as well as standard experimental setups. It is therefore used for fundamental investigations as well as screening type evaluations of different catalysts; most of them related to fuel cells. The two main drawbacks of the TF-RDE method, however, are that i) the determined activities significantly depend on the film homogeneity and although the measurements are easy to perform, it requires experience to obtain meaningful data; that ii) due to limited mass transport the obtained reaction rates - and thus the accessible potential window where kinetic data can be extracted - are limited. Concerning the first drawback, in recent years significant efforts have been spent to develop reliable protocols and best practice guides, ${ }^{[33]}$ which helped to considerably improve the reliability of TF-RDE results. However, mass transport limitation is intrinsic as many reactants of interest (e.g. oxygen) are gaseous and need to be dissolved in the electrolyte, which is limited by their solubility in the electrolyte. As consequence, kinetic activities are in general determined at different potentials (typically $0.9 \mathrm{~V}_{\mathrm{RHE}}$ for the ORR) from what is applied in real systems. In other words, the kinetic ORR activity determined for a fuel cell catalyst is in the range of around 4-5 $\mathrm{mA} \mathrm{cm}_{\text {geo }}^{-2}$, whereas hydrogen fuel

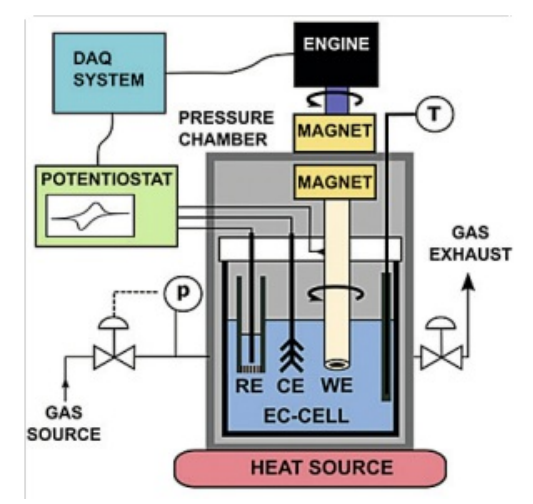
produced with permission of AIP Publishing. ${ }^{[34 b, 35]}$ id measurements up to $160{ }^{\circ} \mathrm{C}$ have been performed. [35] Most important, GDE investigations enable the investigation of catalysts in a straight-forward manner, but at conditions very similar to actual single cell fuel cell measurements. ${ }^{[38]}$ It enables systematic studies of catalysts and electrode layer preparation at high current densities; a topic of strong interest for mobile applications. [39]

\section{Example Studies}

In order to highlight the above described concepts and experimental developments, in the following a few examples of our research with respect to catalyst degradation, particle size and particle proximity effect as well as studies of the solid-liquid interface and its influence on catalytic reactions are given.

\subsection{Degradation of PEM Fuel Cell Catalysts}

The first example of a PEM fuel cell catalyst degradation study is connected to the introduction of IL-TEM. A commercial PEMFC catalyst consisting of Pt nanoparticles supported on a high surface area carbon $(\mathrm{Pt} / \mathrm{C})$ was subjected to an accelerated degradation test (ADT) and its effect on the catalyst was followed on a defined number of Pt nanoparticles. As outlined above the basic degradation mechanisms of a high surface area PEMFC catalyst are i) particle migration and coalescence, ii) metal dissolution, iii) (electrochemical) Ostwald ripening, and iv) particle detachment. Each degradation mechanism is expected to have a particular influence on the size distribution of the nanoparticles; see sketch of size histograms in Fig. 7, where the dotted lines represent the size distribution before and the full line the size distribution after the ADT. a) Pressurized RDE setup b) Gas diffusion electrode (GDE) setup

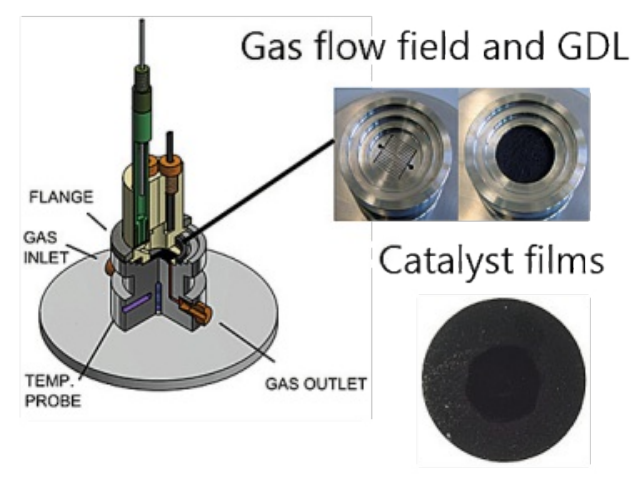

Fig. 6. Sketch of a) pressurized RDE setup and b) gas diffusion electrode (GDE) setup. Figure re- 


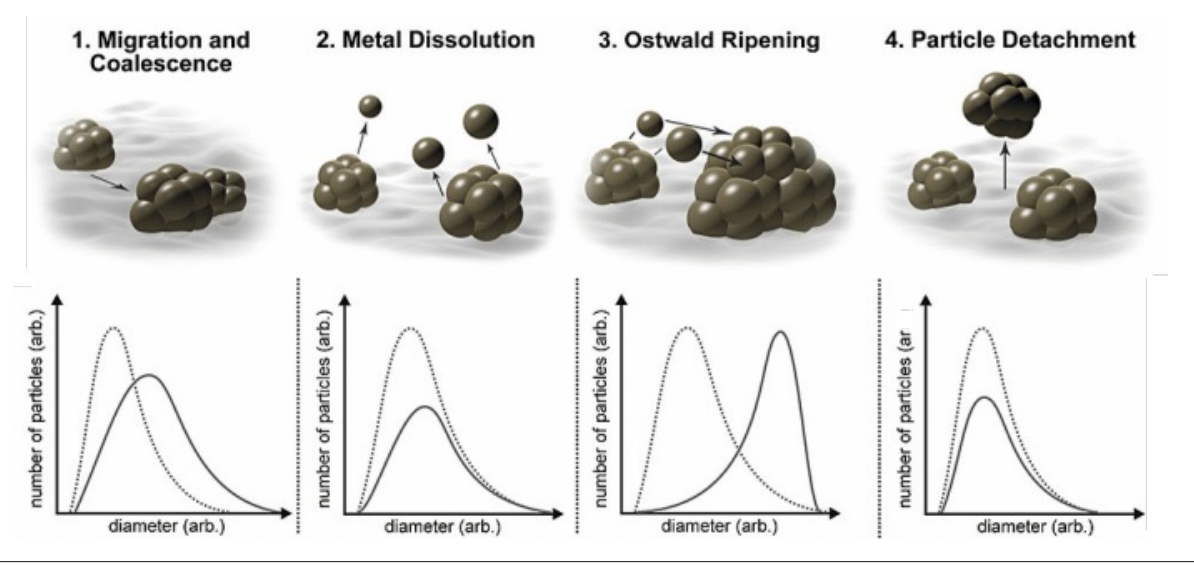

Fig. 7. Sketch of the different high surface area catalyst degradation mechanisms and the expected changes in the particle size distributions. The dotted line represents the respective size histogram before, the full line the size histogram after degradation.

In conventional TEM studies, the different degradation mechanisms are difficult to distinguish. Standard catalysts are not homogeneous and for meaningful analysis several hundred particles need to be counted. Furthermore, some areas (usually with larger agglomerates) cannot be analyzed, posing the question if agglomerate and sintered particles are a result of the ADT, or if they already had been present in the as-prepared catalyst layer. Finally, detecting a change in particle density on the support is not possible either. Thus, metal dissolution and particle detachment for example are extremely difficult to distinguish. In IL-TEM the statistics are based on the tracking of individual particles, see Fig. 8. In the given example, the change in size histograms is characteristic for metal dissolution as well as particle detachment. From the histogram alone, no distinction is possible, although a slight change in the average size could indicate particle dissolution. However, in the series of IL-TEM micrographs it can be seen that the particle density on the carbon support decreases. Furthermore, particles almost always completely disappear from one treatment to the other, but no particle shrinking is observed. Thus IL-TEM unambiguously could show that, under the applied conditions, the main degradation mechanism is particle detachment, not Pt dissolution. The small change in the shape of the size histogram upon applying the ADTs could be a consequence of migration and coalescence, but also a slight preferential detachment of smaller nanoparticles.

In subsequent measurements, we could show that for the ADT used, particle detachment is not always as dominant as in the shown case, but that the degradation mechanism differs for different commercial PEMFC catalysts of the Pt/C type. ${ }^{[24 e]}$

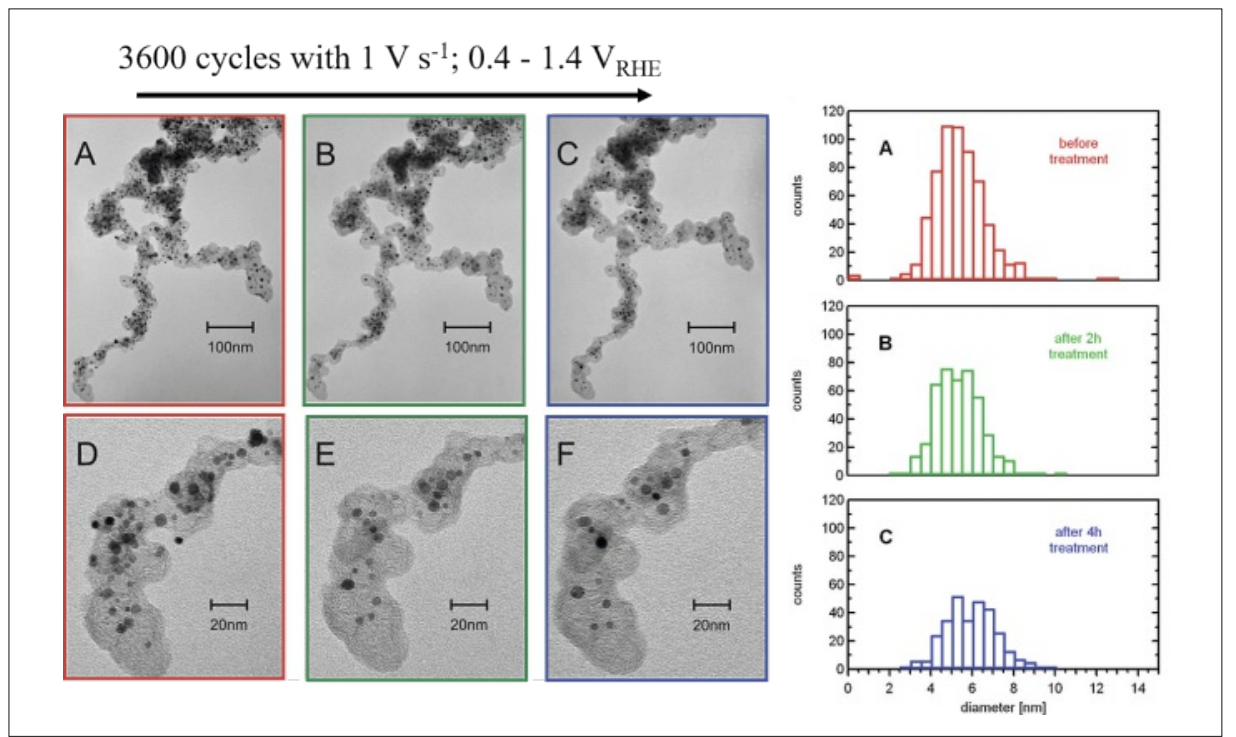

Fig. 8. The influence of two consecutive ADTs on individual Pt nanoparticles (left hand side TEM micrographs) as well as the overall size histogram (right). The ADTs consisted of 1800 cycles each between 0.4 and $1.4 \mathrm{~V}_{\mathrm{RHE}}$ with a scan rate of $1 \mathrm{~V} \mathrm{~s}^{-1}$. Figure reproduced with permission of Elsevier. ${ }^{[24 a]}$

Therefore the fact that in commercial catalyst samples the preparation method is never disclosed severely inhibits the ability to derive general principles concerning the degradation of commercial $\mathrm{Pt} / \mathrm{C}$ catalysts. Applying our tool-box synthesis approach to prepare a series of different $\mathrm{Pt} / \mathrm{C}$ catalysts varying only one property at the time, some more general degradation principles could be derived. For example it could be shown that upon applying an ADT that is suggested to simulate start-stop conditions in PEMFCs ${ }^{[40]}$ (potential cycles between 1.0 and $1.5 \mathrm{~V}_{\mathrm{RHE}}$ with a scan rate of 0.5 $\mathrm{V} \mathrm{s}^{-1}$ ) preferentially carbon corrosion accompanied by particle detachment occurs. On the other hand, an ADT that simulates load cycle conditions (potential steps between 0.6 and $1.0 \mathrm{~V}_{\text {RHE }}$ with a hold time of 3 s) preferentially leads to Pt nanoparticle growth, see Fig. 9. ${ }^{[7 e]}$ An unresolved question is the driving force of the observed $\mathrm{Pt}$ particle migration and the fact that apparently specific particle distances are preferred. ${ }^{[41]}$

\subsection{The Particle Size and the Particle Proximity Effect}

Another long-standing research topic of our group are structure-activity relationships, especially concerning the ORR. This includes the effect of the structure of the catalyst surface, and the particle size effect, as well as more mesoscopic effects of how the particles are arranged on a support, e.g. the particle proximity effect.

The particle size effect on the ORR was first studied using the TF-RDE method by Gasteiger et al..$^{[33 \mathrm{~g}]}$ and Mayrhofer et al..$^{[42]}$ Both studies demonstrated a large decrease in specific ORR activity (SA), i.e. the reaction rate per Pt surface atom, when decreasing the particle size. The results were explained by geometric effects leading to changes in the adsorption strength of $\mathrm{OH}$-intermediates. A later refinement of the TF-RDE measurement procedure by our group, see results in Table 1, however, could demonstrate (using the same commercial $\mathrm{Pt} / \mathrm{C}$ catalysts) that the influence of the particle size is less pronounced than previously reported. Furthermore, modelling trends in the ORR activity expected from different particle geometries and single crystal activity data, it could be shown that the obtained activity trends cannot be explained by sole geometric effects.

A closer look at the data in Table 1 reveals that in the commercial samples that were investigated the particle size and $\mathrm{Pt}$ loading are changed at the same time. This example is a typical case of the problem discussed above, where due to limitations in catalyst synthesis multiple parameters were changed at once. After developing the tool-box synthesis approach, we therefore prepared in a subsequent study two series 
Fig. 9. The influence of different ADTs simulating start-stop conditions (A, B) and load cycle conditions (C, D) on a Pt/C catalyst. Figure reproduced with permission of Elsevier. $\left.{ }^{[7]}\right]$

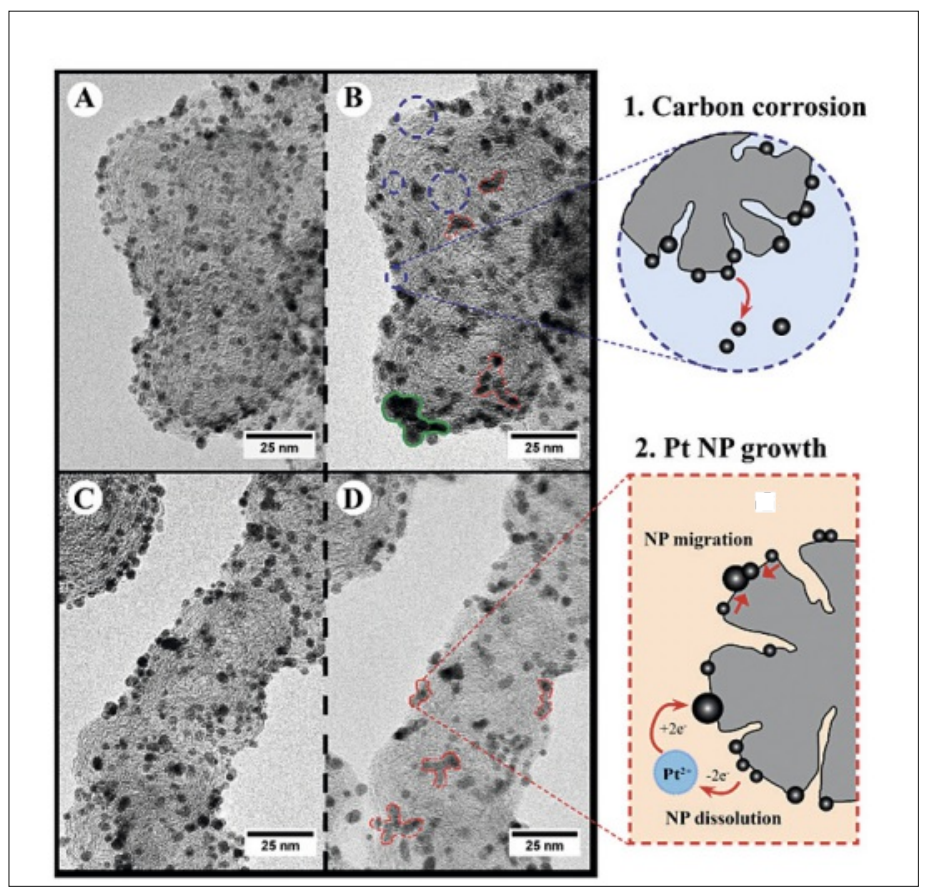

of $\mathrm{Pt} / \mathrm{C}$ catalysts with fixed particle size, but varying Pt loading, see Fig. 10. The two series distinguish themselves only by the choice of carbon support; one carbon support has micropores (Ketjenblack EC300J), whereas the other does not (Vulcan $\mathrm{XC72R}$ ). In each $\mathrm{Pt} / \mathrm{C}$ series the particle size could be kept constant until extremely high Pt loadings, i.e. $>70 \mathrm{wt} . \% \mathrm{Pt}$, as seen by the fact that the determined Pt surface area is independent of the Pt loading. Above $70 \mathrm{wt} . \%$ Pt, particle agglomeration and sintering occurs during catalyst preparation as indicated by the decrease in Pt surface area. The two series of $\mathrm{Pt} / \mathrm{C}$ catalysts were then investigated for their ORR activity and a clear trend became apparent that had been previously described on size-selected Pt nanoparticles deposited onto a planar $\mathrm{GC}$ electrode, i.e. the particle proximity effect of the ORR, see Fig. 11. The particle proximity effect of the ORR describes the fact that the activity of a catalyst not only depends on the properties of the individual particles, but also on the particle assembly. For the ORR this means that the SA sig- nificantly increases for particle assemblies with close particle proximity. Similar observations have been made in noncatalytic studies. For example, it has been argued that very small interparticle separations induce dipole-dipole interactions that lead to an energy transfer between neighboring particles and otherwise insulating assemblies can become semiconducting or even metallic, see ref. [44] and refs. therein. In catalysis, the particle proximity effect has only been studied for a few selected cases and its potential use for optimizing catalytic properties needs to be further exploited.

\subsection{The Solid-Liquid Interface and its Influence on Catalytic Reactions}

Studying the influence of the solid-liquid interface on catalytic reactions is often related to anion adsorption. In early studies concerning structure-activity relationships in electrocatalytic processes, it has been discovered that in half-cell measurements the observed activities are not a consequence of the structure-dependent interaction of the reactants or intermediates with the catalyst, but due to the interaction of the catalyst with the electrolyte. ${ }^{[45]}$ Therefore, for anion adsorption the term 'spectator species' was introduced, highlighting that anions might not have a direct influence of the catalytic processes, but mostly blocks active sites for the reaction. For alkaline electrolytes it could be shown that even non-specifically adsorbed cations influence electrocatalytic reactions. ${ }^{[46]}$

With the introduction of high-temperature PEMFCs where the electrolyte consists of concentrated phosphoric acid embedded into a polybenzimidazole (PBI) polymer, specific anion adsorption gained also practical relevance for applications. To relate anion adsorption with the observed reaction rates, it is interesting to determine the absolute anion coverage at a certain electrode potential. Often this is pursued by constructing 'isotherm-like' plots from cyclic voltammetry, however, the assignment of observed current responses is ambiguous. Even in situ FTIR spectroscopy, see above, only provides qualitative, not quantitative values for the anion coverage. We therefore searched for new approaches to correlate activity data to anion adsorption. By extrapolating data from RDE measurements to potentials where anion adsorption sets in, we could demonstrate a procedure to correlate activity data to the computational modelling of adsorption isotherms. For this we systematically analyzed for the first time RDE ORR data under conditions of high reactant mass transfer conditions, i.e. at diffusion limited conditions. Using the Koutecky-Levich (KL) Eqn. (1) describing the correlation between the measured current density $J$, the kinetic current density $J_{k}$, and the diffusion limited density $J_{d l}$ of a reaction:

$$
\frac{1}{J}=\frac{1}{J_{k}}+\frac{1}{J_{d l}}
$$

we extracted mass-transport free ORR current densities $\left(J_{k l}\right)$ for different concentrations of chloride (a strongly adsorbing an-

Table 1. Summary of the particle size, Pt loading, ECSA, SA, and MA of different commercial Pt/C measured in $0.1 \mathrm{M} \mathrm{HClO} 4$ electrolyte at room temperature conditions. Data taken from ref. [43].

\begin{tabular}{|c|c|c|c|c|}
\hline Particle size [nm] & Pt loading [wt. \%] & $\operatorname{ECSA}\left[\mathbf{m}^{2} \mathbf{g}^{-1}{ }_{\mathrm{pt}}\right]$ & $\mathrm{SA}\left[\mathrm{mAcm}{ }_{\mathrm{Pt}}^{-2}\right]$ & $\mathrm{MA}\left[\mathrm{Amg}_{\mathrm{Pt}}^{-1}\right]$ \\
\hline $1-1.5$ & 19.4 & 128 & 0.43 & 0.55 \\
\hline 2 & 20.1 & 108 & 0.51 & 0.548 \\
\hline $2-3$ & 46 & 76 & 0.49 & 0.374 \\
\hline $4-5$ & 50.6 & 46 & 0.5 & 0.227 \\
\hline 30 & 96 & 13 & 1.2 & 0.151 \\
\hline $\mathrm{Pt}_{\text {bulk }}$ & - & - & 2.1 & - \\
\hline
\end{tabular}


ion) in the electrolyte. The potential dependent values for $J_{k l}$ exhibited clear minima in the potential region where hydrogen underpotential adsorption decreases and anion adsorption sets in (Fig. 12). Combining these measurements with computational modelling, anion adsorption 'isotherms' could be derived. We could extract very accurate data for the Gibbs energies of adsorption of $\mathrm{H}\left(\Delta \mathrm{G}_{\mathrm{H}}^{0}\right)$ and $\mathrm{Cl}\left(\Delta \mathrm{G}_{\mathrm{Cl}}^{0}\right)$, respectively, whereas for the repulsive interactions $\left(\varepsilon_{\mathrm{H}-\mathrm{H}}, \varepsilon_{\mathrm{Cl}-\mathrm{Cl}}\right)$ improved modelling is required (Table 2). In future, we plan to combine such K-L RDE investigations

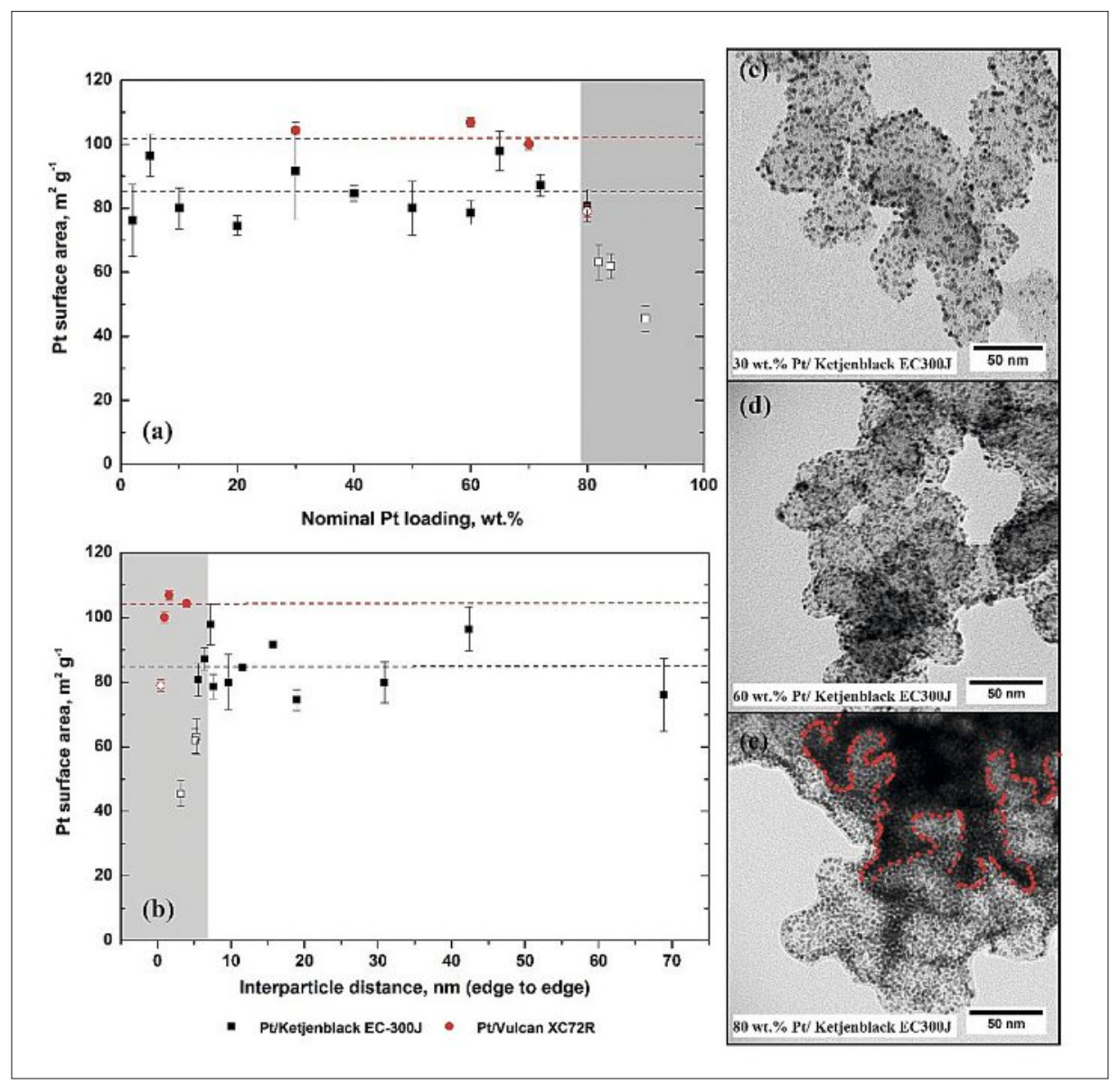

Fig. 10. The ECSA of the catalyst samples as a function of the Pt weight loading (a) and calculated edge to edge interparticle distance (b), respectively. TEM micrographs of the synthesized catalysts are shown at the right-hand side, 30 wt\% (c) 60 wt\% (d) and 80 wt\% (e) Pt/Ketjenblack EC-300J catalysts. Multiple layers of Pt NPs are marked by red dots. Figure reproduced with permission of the Royal Society of Chemistry. ${ }^{[47]}$ a)

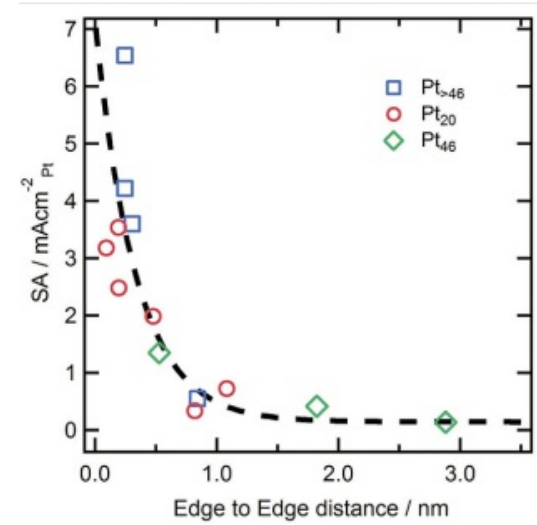

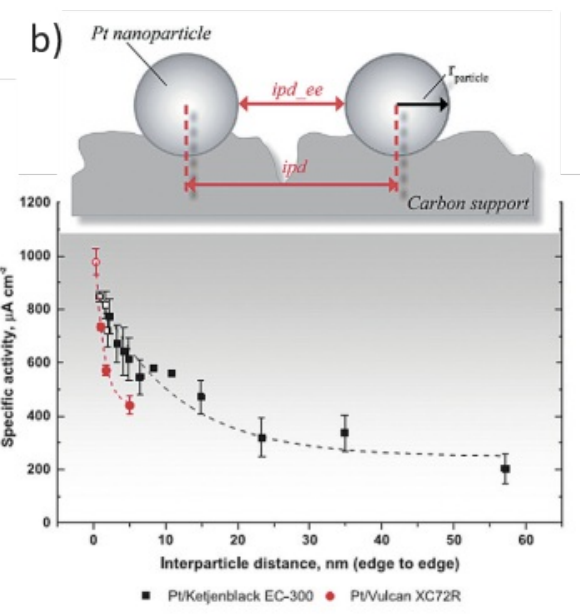

Fig. 11. Specific ORR activities (SA) as function of edge to edge interparticle distance. (a) for size-selected Pt clusters on GC substrate and (b) for carbon supported (Ketjenblack EC-300J and Vulcan XC72R) Pt nanoparticles of same size. Figures reproduced with permission of the Royal Society of Chemistry ${ }^{[47]}$ and SpringerNature. ${ }^{[48]}$ with in situ FTIR spectroscopy to investigate more complex anion adsorption.

\section{Acknowledgements}

The research summarized above would not have been possible without generous research funding as well as excellent co-workers and collaborators. I therefore would like to acknowledge the A.v. Humboldt Foundation (Feodor Lynen Scholarship), German Science Foundation (DFG, Emmy Noether Grant), the Danish Science Foundation (Sapere Aude Forskningsleder Grant, 4M Center, NonPrecious, and DFF 4184-00332 Grants), the European Union Seventh Framework Program (DECORE Project No. 309741 and Cost Action MP0903), as well as support from Umicore, the Villum Foundation, and Toyota Central R\&D Labs. I would like to thank all my former and present co-workers in Berkeley, Munich, Copenhagen, and Bern as well as all my collaborators.

Received: February 20, 2018

[1] G. Ertl, Angew. Chem. Int. Ed. 2008, 47, 3524.

[2] I. Chorkendorff, J. W. Niemantsverdriet, 'Concepts of modern catalysis and kinetics', WILEY-VCH, Weinheim, 2007.

[3] G. J. Hutchings, J. C. Védrine, in 'Basic Principles in Applied Catalysis', Ed. M. Baerns, Springer Berlin Heidelberg, Berlin, Heidelberg, 2004, p. 215.

[4] M. Raney, Ind. Eng. Chem. 1940, 32, 1199.

[5] O. Deutschmann, H. Knözinger, K. Kochloefl, T. Turek, in 'Ullmann's Encyclopedia of Industrial Chemistry', Wiley, 2009.

[6] R. Strobel, W. J. Stark, L. Madler, S. E. Pratsinis, A. Baiker, J. Catal. 2003, 213, 296.

[7] a) J. Speder, L. Altmann, M. Roefzaad, M Baumer, J. J. K. Kirkensgaard, K. Mortensen, M Arenz, Phys. Chem. Chem. Phys. 2013, 15, 3602; b) A. Zana, T. Vosch, M. Arenz, Electrochim. Acta 2016, 197, 221; c) J. Speder, A. Zana, M. Arenz, Catal. Today 2016, 262, 82; d) J. Speder, I. Spanos, A. Zana, J. J. K. Kirkensgaard, K. Mortensen, L. Altmann, M. Baumer, M. Arenz, Surf. Sci. 2015, 631, 278; e) J. Speder, A. Zana, I. Spanos, J. J. K. Kirkensgaard, K. Mortensen, M. Hanzlik, M. Arenz, J. Power Sources 2014, 261, 14.

[8] Y. Wang, J. W. Ren, K. Deng, L. L. Gui, Y. Q. Tang, Chem. Mater. 2000, 12, 1622.

[9] S. Neumann, S. Grotheer, J. Tielke, I. Schrader, J. Quinson, A. Zana, M. Oezaslan, M. Arenz, S. Kunz, J. Mater. Chem. A 2017, 5, 6140.

[10] Y. Wang, J. L. Zhang, X. D. Wang, J. W. Ren, B. J. Zuo, Y. Q. Tang, Top. Catal. 2005, 35, 35.

[11] a) I. Schrader, J. Warneke, S. Neumann, S. Grotheer, A. A. Swane, J. J. K. Kirkensgaard, M. Arenz, S. Kunz, J. Phys. Chem. C 2015, 119, 17655; b) L. Kacenauskaite, J. Quinson, H. Schultz, J. J. K. Kirkensgaard, S. Kunz, T. Vosch, M. Arenz, Chemnanomat. 2017, 3, 89.

[12] E. Morsbach, J. Speder, M. Arenz, E. Brauns, W. Lang, S. Kunz, M. Baumer, Langmuir 2014, 30, 5564 .

[13] S. Kunz, P. Schreiber, M. Ludwig, M. M. Maturi, O. Ackermann, M. Tschurl, U. Heiz, Phys. Chem. Chem. Phys. 2013, 15, 19253.

[14] I. Schrader, J. Warneke, J. Backenkohler, S. Kunz, J. Am. Chem. Soc. 2015, 137, 905.

[15] J. Y. Park, C. Aliaga, J. R. Renzas, H. Lee, G. A Somorjai, Catal. Lett. 2009, 129, 1.

[16] a) S. Kunz, E. Iglesia, J. Phys. Chem. C 2014, 118, 7468; b) J. C. Meier, C. Galeano, I. Katsounaros, A. A. Topalov, A. Kostka, F. Schuth, K. J. J. Mayrhofer, ACS Catal. 2012, 2, 832. 
Fig. 12. Plot of the mass transport free ORR current densities $\left(\mathrm{J}_{\mathrm{k}}\right)$ extracted from the $K-L$ equation (Eqn. 1) in 0.01 $\mathrm{M}, 0.1 \mathrm{M}$ and $1 \mathrm{M}$ $\mathrm{HCl}$ electrolyte solution. The insert is a magnification of the measurements at higher electrolyte concentrations. The shaded area represents the uncertainty in the measurements. Figures reproduced with permission of the American Chemical Society. [49]

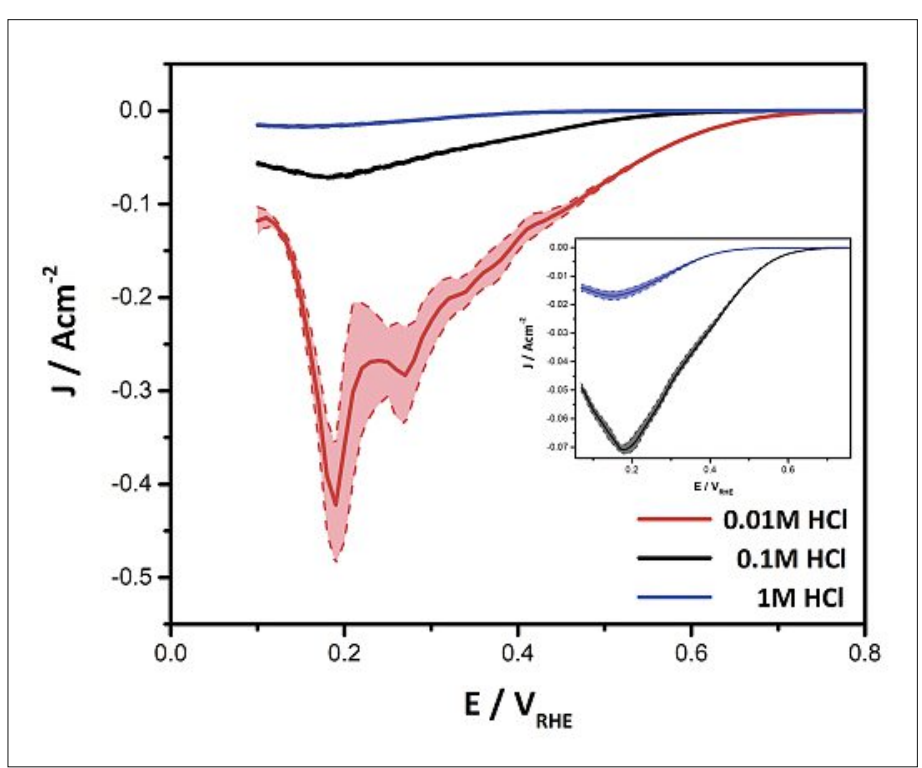

Table 2. Comparison between parameters fitted according to site blocking model of anion adsorption and literature values. Data taken from ref. [49].

\begin{tabular}{|l|l|l|}
\hline Parameter & fit & Literature value \\
\hline$\Delta \mathrm{G}_{\mathrm{H}}^{0}[\mathrm{eV}]$ & -0.18 & $-0.2,^{[50]}-0.1^{[51]}$ \\
\hline$\varepsilon_{\mathrm{H}-\mathrm{H}}[\mathrm{eV}]$ & 0.12 & $0.15^{[50]}$ \\
\hline$\Delta \mathrm{G}_{\mathrm{Cl}}^{0}[\mathrm{eV}]$ & -1.42 & $-1.31^{[52]}$ \\
\hline$\varepsilon_{\mathrm{Cl}-\mathrm{Cl}}[\mathrm{eV}]$ & 0.18 & $0.7^{[53]}$ \\
\hline$j_{0}\left[\mathrm{~mA} \mathrm{~cm}{ }^{-2}\right]$ & 240 & $96^{[53]}$ (value for $\left.\mathrm{Pt}(111)\right)$ \\
\hline
\end{tabular}

[17] a) M. Inaba, J. Quinson, J. R. Bucher, M. Arenz, J. Vis. Exp. 2018, 133, e57105; b) I. Spanos, K Dideriksen, J. J. K. Kirkensgaard, S. Jelavic, M. Arenz, Phys. Chem. Chem. Phys. 2015, 17, 28044.

[18] M. Inaba, J. Quinson, M. Arenz, J. Power Sources 2017, 353, 19 .

[19] S. Kunz, M. Arenz, J. Quinson, L. Kacenauskaite, 2017, PCT application.

[20] a) M. K. Debe, A. J. Steinbach, G. D. Vernstrom, S. M. Hendricks, M. J. Kurkowski, R. T Atanasoski, P. Kadera, D. A. Stevens, R. J. Sanderson, E. Marvel, J. R. Dahn, J. Electrochem. Soc. 2011, 158, B910; b) M. K. Debe, A. K. Schmoeckel, G. D. Vernstrorn, R. Atanasoski, J. Power Sources 2006, 161, 1002.

[21] D. van der Vliet, C. Wang, M. Debe, R. Atanasoski, N. M. Markovic, V. R. Stamenkovic, Electrochim. Acta 2011, 56, 8695.

[22] S. Henning, H. Ishikawa, L. Kuhn, J. Herranz, E. Muller, A. Eychmuller, T. J. Schmidt, Angew. Chem. Int. Ed. 2017, 56, 10707.

[23] NordicElectrochemistry_ApS, www.nordicec.com.

[24] a) K. J. J. Mayrhofer, J. C. Meier, S. J. Ashton, G K. H. Wiberg, F. Kraus, M. Hanzlik, M. Arenz, Electrochem. Commun. 2008, 10, 1144; b) K. J. J. Mayrhofer, S. J. Ashton, J. C. Meier, G. K. H. Wiberg, M. Hanzlik, M. Arenz, J. Power Sources 2008, 185, 734; c) K. Hartl, M. Hanzlik, M. Arenz, Energ. Environ. Sci. 2011, 4, 234; d) K. Schlogl, K. J. J. Mayrhofer, M. Hanzlik, M. Arenz, J. Electroanal. Chem. 2011, 662 , 355; e) K. Schlog1, M. Hanzlik, M. Arenz, J. Electrochem. Soc. 2012, 159, B677; f) A. Zana, J. Speder, M. Roefzaad, L. Altmann, M. Baumer, M. Arenz, J. Electrochem. Soc. 2013, 160, F608.

[25] a) N. Hodnik, M. Zorko, M. Bele, S. Hocevar, M. Gaberscek, J. Phys. Chem. C 2012, 116,
[29] M. Nesselberger, S. J. Ashton, G. K. H. Wiberg, M. Arenz, Rev. Sci. Instrum. 2013, 84, 074103.
[30] M. Nesselberger, M. Arenz, Chemcatchem 2016, 8,1125 .

[31] H. Miyake, S. Ye, M. Osawa, Electrochem. Commun. 2002, 4, 973.

[32] T. J. Schmidt, H. A. Gasteiger, G. D. Stab, P. M Urban, D. M. Kolb, R. J. Behm, J. Electrochem. Soc. 1998, 145, 2354.

[33] a) S. S. Kocha, K. Shinozaki, J. W. Zack, D. J. Myers, N. N. Kariuki, T. Nowicki, V. Stamenkovic, Y. J. Kang, D. G. Li, D. Papageorgopoulos, Electrocatalysis 2017, 8, 366; b) K. Shinozaki, J. W. Zack, S. Pylypenko, R. M. Richards, B. S. Pivovar, S. S. Kocha, Int. J. Hydrogen Energ. 2015, 40, 16820; c) K. Shinozaki, J. W. Zack, S. Pylypenko, B. S. Pivovar, S. S. Kocha, J. Electrochem. Soc. 2015, 162, F1384; d) K. Shinozaki, J. W. Zack, R. M. Richards, B. S. Pivovar, S. S. Kocha, J Electrochem. Soc. 2015 162, F1144; e) Y. Garsany, I. L. Singer, K. E. Swider-Lyons, J. Electroanal. Chem. 2011, 662, 396; f) K. Ke, K. Hiroshima, Y. Kamitaka, T. Hatanaka, Y. Morimoto, Electrochim. Acta 2012, 72, 120; g) H. A. Gasteiger, S. S. Kocha, B. Sompalli, F. T. Wagner, Appl. Catal. B-Environ. 2005, 56, 9; h) K. J. J. Mayrhofer, D. Strmcnik, B. B. Blizanac, V. Stamenkovic, M. Arenz, N. M. Markovic, Electrochim. Acta 2008, 53, 3181.

[34] a) M. J. Fleige, G. K. H. Wiberg, M. Arenz, Rev. Sci. Instrum. 2015, 86, 064101; b) G. K. H. Wiberg, M. J. Fleige, M. Arenz, Rev. Sci. Instrum. 2014, 85, 085105.

[35] G. K. H. Wiberg, M. Fleige, M. Arenz, Rev. Sci. Instrum. 2015, 86, 024102.

[36] M. Fleige, K. Holst-Olesen, G. K. H. Wiberg, M Arenz, Electrochim. Acta 2016, 209, 399.

[37] B. A. Pinaud, A. Bonakdarpour, L. Daniel, J. Sharman, D. P. Wilkinson, J. Electrochem. Soc. 2017, 164, F321.

[38] M. Inaba, A. Westergard Jensen, G. W. Sievers, M. Escudero-Escribano, A. Zana, M. Arenz, Ener Environ. Sci. 2018, doi:10.1039/C8EE00010K

[39] A. Kongkanand, M. F. Mathias, J. Phys. Chem. Lett. 2016, 7, 1127.

[40] A. Ohma, K. Shinohara, A. Iiyama, T. Yoshida, A. Daimaru, ECS Trans. 2011, 41, 775.

[41] K. Hartl, M. Nesselberger, K. J. J. Mayrhofer, S. Kunz, F. F. Schweinberger, G. Kwon, M. Hanzlik, U. Heiz, M. Arenz, Electrochim. Acta 2010, 56, 810 .

[42] K. J. J. Mayrhofer, B. B. Blizanac, M. Arenz, V. R. Stamenkovic, P. N. Ross, N. M. Markovic, $J$ Phys. Chem. B 2005, 109, 14433.

[43] M. Nesselberger, S. Ashton, J. C. Meier, I Katsounaros, K. J. J. Mayrhofer, M. Arenz, J. Am. Chem. Soc. 2011, 133, 17428.

[44] C. B. Murray, C. R. Kagan, M. G. Bawendi, Annu. Rev. Mater. Sci. 2000, 30, 545.

[45] N. M. Markovic, P. N. Ross, Surf. Sci. Rep. 2002 45, 121.

[46] P. P. Lopes, D. Strmcnik, J. S. Jirkovsky, J. G. Connell, V. Stamenkovic, N. Markovic, Catal. Today 2016, 262, 41.

[47] J. Speder, L. Altmann, M. Baumer, J. J. K. Kirkensgaard, K. Mortensen, M. Arenz, RSC Adv. 2014, 4, 14971.

[48] M. Nesselberger, M. Roefzaad, R. F. Hamou, P. U. Biedermann, F. F. Schweinberger, S. Kunz, K. Schloegl, G. K. H. Wiberg, S. Ashton, U. Heiz, K. J. J. Mayrhofer, M. Arenz, Nat. Mater. 2013, 12, 919.

[49] A. Zana, G. K. H. Wiberg, Y. J. Deng, T. Ostergaard, J. Rossmeisl, M. Arenz, ACS Appl. Mater. Inter. 2017, 9, 38176.

[50] G. S. Karlberg, T. F. Jaramillo, E. Skulason, J. Rossmeisl, T. Bligaard, J. K. Norskov, Phys. Rev. Lett. 2007, 99, 126101.

[51] J. K. Norskov, T. Bligaard, A. Logadottir, J. R. Kitchin, J. G. Chen, S. Pandelov, J. K. Norskov, J. Electrochem. Soc. 2005, 152, J23.

[52] F. Gossenberger, T. Roman, A. Gross, Surf. Sci. 2015, 631, 17 .

[53] J. Rossmeisl, G. S. Karlberg, T. Jaramillo, J. K. Norskov, Faraday Disc. 2008, 140, 337. 